Journal of Engineering and Applied Sciences 14 (Special Issue 6): 9214-9218, 2019

ISSN: 1816-949X

(C) Medwell Journals, 2019

\title{
Elucidation of Weak D Phenotype among Malaysian Blood Donors using Molecular Basis
}

\author{
${ }^{1}$ Evana Kamarudin, ${ }^{1}$ Mohd Khairul Amran Mohammad, ${ }^{1}$ Tressa Gadung Saptu and \\ ${ }^{2}$ Rozi Hanisa Musa \\ ${ }^{1}$ Department of Medical Laboratory Technology, Faculty of Health Sciences, \\ Universiti Teknologi MARA Selangor, Puncak Alam Campus, \\ 42300 Kuala Selangor, Selangor, Malaysia \\ ${ }^{2}$ Immunohematology Division, National Blood Center of Malaysia, \\ Jalan Tun Razak, 50400 Kuala Lumpur, Malaysia
}

\begin{abstract}
The Rh blood group system is the most complex, polymorphic and immunogenic protein-based blood group system in humans. Some individuals produce a weak expression of D antigen on RBCs surface as a result of missense RHD mutations and amino acid substitutions that lead to distinct serologic phenotypes and anti-D immunization. This study aimed to elucidate the molecular genetic basis of weak D phenotypes in Malaysian blood donors with multiracial population. A total of 183 Rh-negative blood samples were phenotyped for D, C, c, E and e antigens. Weak D samples that identified by Indirect Antiglobulin Test (IAT) were amplified by polymerase chain reaction-sequence specific primers for weak $D$ type determination. A total of 183 multiracial Rh-negative donors were reviewed, consisting of 88 (48.1\%) Indians, 56 (30.6\%) Malays, 23 (12.6\%) Chinese and $16(8.7 \%)$ other minority ethnics. Four samples were found to be positive for weak D phenotype using IAT. Of these four samples, two samples were reported with weak D type 15. Both samples were from Chinese blood donor with $D^{\text {weak }} C$ cee and $D^{\text {weak }}$ ccEe phenotype, respectively. This study provide the first database of a molecular basis of weak D in Malaysian blood donors which improved the understanding of molecular mechanisms underlying D antigen expression in Malaysian population. Thus, increase the transfusion safety in highly racial mixed population.
\end{abstract}

Key words: Rh negative, weak D phenotype, Malaysian blood donors, Malaysian population, polymerase chain, transfusion safety

\section{INTRODUCTION}

The Rh blood group system is the most complex, polymorphic and immunogenic protein-based blood group system in humans (Zacarias et al., 2016). It represents the largest number of antigens out of the 36 known blood group systems which comprised of 54 antigens numbered RH1 to RH61 and more than 200 alleles for RHD gene alone (Flegel, 2011; ISBT., 2016). The Rh antigen are encoded by two highly homologous genes (RHD and $\mathrm{RHCE}$ ) in Rh locus that localized in chromosome 1p34.4-p36.1. These genes are placed in opposite orientations and share more than $90 \%$ homology of all introns and coding region (Chen et al., 2016).

The $\mathrm{D}$ blood group antigen is a clinically significant protein of Rh system due to its association in haemolytic disease of newborn, haemolytic transfusion reactions and autoimmune haemolytic anaemia (Cruz et al., 2012).
The RHD gene encoded the $\mathrm{RhD}$ protein that expressing the D antigen while the RHCE gene encoded for $\mathrm{RhCcEe}$ protein that expressing $\mathrm{Ce}, \mathrm{CE}$, ce or $\mathrm{cE}$ antigens. In transfusion medicine, each individual is clinically classified as Rh-positive or Rh-negative depending on the presence or absence of the $\mathrm{D}$ antigen on the Red Blood Cell (RBC) membrane. Rh-positive was determined by expression of $\mathrm{D}$ antigen that encoded by normal and functional RHD gene intact with RHCE gene on a single locus. Meanwhile, Rh-negative indicates total absence of $\mathrm{D}$ antigen that commonly caused by complete absence of the $\mathrm{RhD}$ protein which clarifying the high immunogenicity of D. Genetic background of Rh-negative explained the difference among the majors human population. The prevalence of Rh-negative phenotype was estimated $<3 \%$ in Asians, $15-17 \%$ in Caucasians and $5 \%$ in Black Africans (Cruz et al., 2012). The RHD gene polymorphism contributes to phenotypic polymorphism

Corresponding Author: Evana Kamarudin, Department of Medical Laboratory Technology, Faculty of Health Sciences, Universiti Teknologi MARA Selangor, Puncak Alam Campus, 42300 Kuala Selangor, Selangor, Malaysia 
$\mathrm{D}$ variants including weak $\mathrm{D}, \mathrm{Del}$ and partial $\mathrm{D}$ (Wafi et al., 2016). A serologic weak D phenotype is defined by reduced $\mathrm{RhD}$ expression on RBCs membrane compared to its expression in the vast majority of Rh-positive individual (Chen et al., 2012). The reactivity of weak D RBCs with anti-D giving no or $>2+$ reaction $(<2+)$ in initial testing and it can only react with IgG anti-D by Indirect Antiglobulin Test (IAT) (Sandler and Flegel, 2015). Weak expression of $D$ antigen is caused by missense RHD mutations and amino acid substitutions of weak $D$ types are positioned in intracellular and transmembraneous protein segments and clustered in four regions of the $\mathrm{Rh}$ protein (Chen et al., 2012). The prevalence of weak $\mathrm{D}$ antigen ranges from $0.2-1 \%$ and these incidence occurred varies in different ethnic groups, populations and geographical locales (Chen et al., 2012). It was demonstrated that persons with weak D type 1, 2, $3,4.0$ or 4.1 have never been reported to cause formation of alloanti-D. However, it was documented that certain other types of weak D including weak D type 4.2, DAR, type $11,15,21$ and 57 have been reported to be associated with alloimmunization (Sandler et al., 2015).

In fact, some weak $\mathrm{D}$ are not detected by serologic techniques. This become a worldwide concern in blood transfusion due to its potential to cause anti-D alloimmunisation when donors with certain weak $\mathrm{D}$ types being transfuse to Rh-negative patient. Considering the risk of forming alloanti-D following blood transfusion and the diverse ethnicity of Malaysian, this study aims to elucidate the molecular genetic basis of weak D phenotypes in Malaysian blood donor, a representative sample of a genetically diverse population due to its high degree of miscegenation.

\section{MATERIALS AND METHODS}

Blood specimen collection: This study was carried out in accordance with the standards recommended by Medical Research and Ethics Committee (MREC), Ministry of Health Malaysia (NMMR-15-1622-25347). A total of 183 Rh-negative unrelated blood donor's samples were collected in Ethylenediaminetetraacetate (EDTA) tubes from National Blood Centre (NBC) between Mar. to Jun 2016.

Rhesus phenotyping: The Rhesus phenotype for C, c, D, $\mathrm{E}$ and e of all donor's RBCs samples were determined by standard serologic protocols. The commercial antibody reagents used for Rhesus typing were from CSL (Australia), Millipore (United Kingdom) and Bio-Rad (Switzerland). Samples that showed weak agglutination with anti-D sera $(<2+)$ were determined for weak D phenotype using an IAT.
DNA extraction and quantification: Genomic DNA was isolated from peripheral blood cells using the QIAamp DNA Blood Mini Kit (Qiagen, Hilden, Germany) following the manufacturer's instructions. DNA concentration and purity were determined using biophotometer (Eppendorf, USA) and purity index in between 1.7-2.0.

PCR-SSP genotyping for weak D types: For molecular analysis, BAGene PCR-SSP kit (BAG Health Care, Lich, Germany) was used to identify specific weak D allele according to the manufacture's instruction. BAGene PCR-SSP for weak D kit was designed to identify weak D type $1,1.1,2,3,4.0$ or $4.1,4.2,5,11,15,17$ and 20 . PCR strip for weak D genotyping consisted of 8 reaction mixture. Reaction 1 consists of multiplex-PCR reaction targeting weak D allele type 1 (150 bp) and 1.1 (150 and $250 \mathrm{bp}$ ). Reaction 2 consists of multiplex-PCR reaction targeting weak D allele type $2(126 \mathrm{bp})$. Reaction 3 consists of multiplex-PCR reaction targeting weak D allele type 3 ( $165 \mathrm{bp}$ ). Reaction 4 consists of multiplex-PCR reactions targeting weak D allele type 4.0 or 4.1 and weak D allele type $4.2(101 \mathrm{bp})$. Reaction 5 consists of multiplex-PCR reaction targeting weak $\mathrm{D}$ allele type 4.2 (130 bp) and weak D allele type 17 (83 bp). Reaction 6 consists of multiplex-PCR reaction targeting weak $\mathrm{D}$ allele type 5 (112 bp). Reaction 7 consists of multiplex-PCR reaction targeting weak $D$ allele type 11 (198 bp) and weak D allele type $17(83 \mathrm{bp})$. Reaction 8 consists of multiplex-PCR reaction targeting weak $D$ allele type 15 (153 bp). The internal control product will appeared in all lanes at $434 \mathrm{bp}$. PCR products were visualized in $2 \%$ agarose gel.

\section{RESULTS AND DISCUSSION}

Serologic test results: The prevalence of Rh-negative blood group differs considerably among ethnics groups and populations. The frequency of Rh-negative was estimated $>3 \%$ in Asians, $15-17 \%$ in Caucasians and $5 \%$ in Black Africans (Cruz et al., 2012). In this study, the distribution of Rh-negative phenotype was carried out among Malaysian blood donor which represent highly racial mixed population. Malaysia, a multiracial population comprising of three major races (Malays, Chinese and Indian) and other ethnic groups. Thus, this study aimed to investigate the distribution of Rh-negative phenotypes among the ethnic groups in the Malaysian population.

Between the periods of Mar to June 2016, a total of 183 Rh-negative blood donors were collected at NBC (Table 1). Of these blood donors, 88 (48.1\%) Indians, 56 (30.6\%) Malays, $23(12.6 \%)$ Chinese and $16(8.7 \%)$ other 
Table 1: The distribution of different Rh phenotypes among 183 blood donors of various races at NBC, Kuala Lumpur, Malaysia

\begin{tabular}{llllll}
\hline & \multicolumn{5}{l}{ Races } \\
\cline { 3 - 5 } Rh phenotype & Frequency $(\mathrm{n}=183)$ & $\mathrm{I}$ & $\mathrm{M}$ & $\mathrm{C}$ & $\mathrm{O}$ \\
\hline dccee & $162(88.52 \%)$ & 85 & 44 & 17 & 16 \\
dCCee & $12(6.56 \%)$ & 2 & 8 & 2 & 0 \\
dCcee & $4(2.18 \%)$ & 0 & 2 & 2 & 0 \\
dCcEe & $1(0.55 \%)$ & 0 & 1 & 0 & 0 \\
$\mathrm{D}^{\text {weak }}$ Ccee & $2(1.09 \%)$ & 1 & 0 & 1 & 0 \\
$\mathrm{D}^{\text {weak }}$ ccEe & $1(0.55 \%)$ & 0 & 0 & 1 & 0 \\
$\mathrm{D}^{\text {weak }}$ ccee & $1(0.55 \%)$ & 0 & 1 & 0 & 0 \\
TOTAL & $183(100 \%)$ & 88 & 56 & 23 & 16 \\
\hline
\end{tabular}

I: Indian, M: Malay, C: Chinese, O: Others

minor ethnic groups. The commonest phenotype recorded was found to be dccee, $162(88.52 \%)$. This was observed in $85(96.6 \%)$ Indian, $44(78.6 \%)$ Malays and $17(73.9 \%)$ in Chinese. The remaining $16(100 \%)$ consisted of donor from minor ethnic groups. The prevalence of dccee phenotype in Rh-negative blood donor in Malaysia reported by Kyaw et al. (2012) was found to be 750 out of 911 Rh-negative blood donor between the period of January 2003 and September 2008, showed nearly similar with present findings. Out of 750 dccee phenotype, $91.5 \%$ Indian, $74.6 \%$ Malays, $55.5 \%$ Chinese and $92.5 \%$ others (minor ethnic groups). Meanwhile, Rh-negative blood donors with $\mathrm{C}$ and or $\mathrm{E}$ antigens including $\mathrm{CCee}, \mathrm{Ccee}$ and $\mathrm{CcEe}$ were reported to be $6.56 \%(12 / 183), 2.18 \%(4 / 183)$ and $0.55 \%(1 / 183)$, respectively. No dccEe phenotype was recorded in this study. Malays showed the most prevalence Rh-negative with $\mathrm{C}$ and or $\mathrm{E}$ antigens 8 compared to Chinese 4 and Indian 2 (Table 1).

Weak D phenotype is defined by weak expression of $\mathrm{RhD}$ antigen on $\mathrm{RBCs}$ membrane which result in reduced reactivity of anti-D, giving no or $>2+$ reaction $(<2+)$. This type of phenotypic polymorphism $D$ variants can only react with IgG anti-D by Indirect Antiglobulin Test (IAT) giving moderate to strong agglutination (Chen et al., 2012; Sandler et al., 2015). Out of 183 Rh-negative blood samples, 4 samples were found to be positive for weak D phenotype. The two samples that positive for weak D phenotype were present with Ccee and ccEe phenotypes. The incidence of weak D worldwide range from $0.2-1 \%$ as reported by Gupta et al. (2016) and majority are associated with weak D type 1-3. While the molecular genetic basis of weak D are well investigated in Caucasian and other population to the best of our knowledge, there is no data on weak $\mathrm{D}$ distribution among Asian population.

Molecular analysis of weak D type by PCR-SSP: Genomic DNA of the four weak D phenotypes samples were subjected to commercially available PCR-SSP for detection of weak D types. Of these four samples, two samples were reported with weak D type 15 . Both samples were from
Table 2: Weak D phenotype and associated types in Malay sian population positive with IAT $(n=4)$

\begin{tabular}{llll}
\hline Sample & Weak D phenotype & Race & Weak type by PCR-SSP \\
\hline 1 & $\mathrm{D}^{\text {weak }} \mathrm{Ccee}$ & $\mathrm{C}$ & Type 15 \\
2 & $\mathrm{D}^{\text {weak }} \mathrm{ccee}$ & $\mathrm{I}$ & No type detected \\
3 & $\mathrm{D}^{\text {weak }} \mathrm{ccEe}$ & $\mathrm{C}$ & Type 15 \\
4 & $\mathrm{D}^{\text {weak }} \mathrm{Ccee}$ & $\mathrm{M}$ & No type detected \\
\hline
\end{tabular}

I:Indian, M:Malay, C: Chinese

Chinese blood donor with $\mathrm{D}^{\text {weak }} \mathrm{Ccee}$ and $\mathrm{D}^{\text {weak }} \mathrm{ccEe}$ phenotype, respectively. Wagner et al. (1999), Virk and Sandler (2015) have reported that weak D type 15 has associated with cDE haplotype. In addition, Shao et al. (2012) also have similarly reported that weak D type 15 was observed with cEe phenotype. While present study reported that weak $\mathrm{D}$ type 15 was observed with the present of Ccee and ccEe phenotype. In such case, weak $D$ type 15 was assumed has independently related with $C$ and or $\mathrm{E}$ antigen and molecular genotyping for weak $\mathrm{D}$ was optional for weak D phenotype present with either $\mathrm{C}$ and or E antigen (Table 2).

Figure 1 shows analysis of weak D type present in individual with weak D phenotype using PCR-SSP. The PCR products were analyzed by agarose gel electrophoresis. Weak D type 15 was observed in lane 9 with product size of $153 \mathrm{bp}$. In all lanes (lane 2-9), a $434 \mathrm{bp}$ fragments was amplified representing the internal control. M, 100 bp ladder marker.

Distribution of weak D among countries showed difference of occurrences. Based on that reason, previous study recommended that determination of molecular basis of weak D should be applied in own country. Studies on molecular basis of weak D in Europe have underlined three major occurrence weak D types which are weak D type 1-3. Even that the occurrences of the three major weak D types showed difference in frequency among countries in Europe. In Portugal, weak D type 2 showed the most common among the three major weak D types. Weak D type 1 was the most common in Northern and Southwestern Germany while weak D type 3 was the common Tyrol, Austria. In African, weak D type 4 was detected. Findings of type weak D in Europe and African were differed from the finding of present study which recall back that distribution of types of weak $\mathrm{D}$ were showed difference among countries.

Similar finding of weak D type 15 also can be observed in Chinese population of China. Shao et al. (2012) firstly observed weak D type 15 in Chinese individual. Moreover, Yan et al. (2007) found that weak D type 15 was highly observed in Chinese individuals. Currently, weak D type 15 found to be predominant type of weak D in Chinese individuals specifically in Zhejiang Hans population (He et al., 2015). Therefore, weak D type 15 known to be the type of weak D that commonly can be 


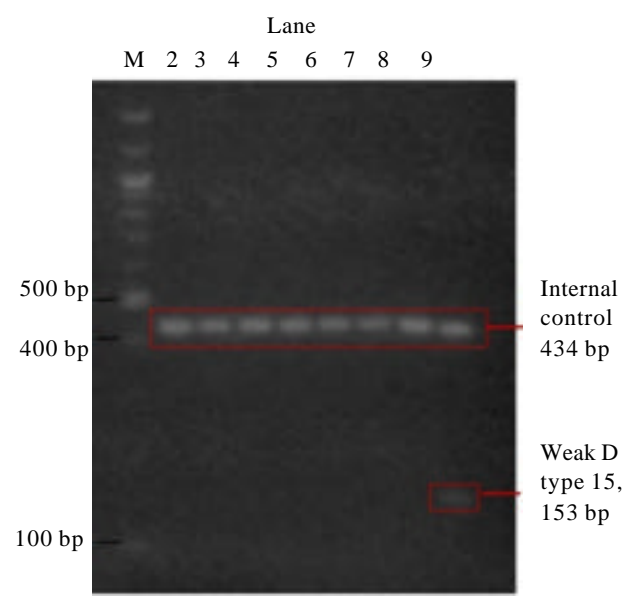

Fig. 1: Analysis of weak D type

observed in Chinese individuals. Besides, weak D type 15 also was identified from Korean blood donors (Luettringhaus et al., 2006). Other than that similar finding also was observed in Taiwanese (Lin et al., 2003). Based on previous studies in Asia and also present study, weak D type 15 was assumed to represent the common type of weak D that can be observed in Malaysian population.

\section{CONCLUSION}

We investigated the molecular mechanism of weak D phenotype among Rh-negative in Malaysian population. Out of 183 Rh-negative blood donors, 4 samples were positive for weak D phenotype by IAT. However, among these 4, 2 samples were reported with weak D type 15 based on amplification using PCR-SSP. Both samples were from Chinese blood donor with $D^{\text {weak }} \mathrm{Ccee}$ and $\mathrm{D}^{\text {weak }} \mathrm{ccEe}$ phenotype, respectively. This study added to the understanding of molecular mechanisms underlying D antigen expression in Malaysian population and provides useful information for the medical representative to adopt suitable genotyping approaches in routine test. It is recommended to discover more on weak $\mathrm{D}$ type in Malaysia in other to effectively utilize the negative blood and to avoid unnecessary Anti D prophylaxis.

\section{ACKNOWLEDGEMENTS}

The researchers gratefully acknowledge the encouragement and financial support given by UiTM Research Management Center (RAGS:600-RMI/RAGS 5/3 (37/2014)) for funding the study. The researchers deeply appreciate Department of Medical Laboratory Technology, Department of Postgraduate Study, Faculty of Health Sciences, UiTM Selangor, Puncak Alam Campus and the entire staff of Immunohaematology Department, National Blood Center, Kuala Lumpur for their corporation and providing facilities throughout this study.

\section{REFERENCES}

Chen, D.P., C.F. Sun, H.C. Ning, W.T. Wang and C.P. Tseng, 2016. Comprehensive analysis of RHD splicing transcripts reveals the molecular basis for the weak anti-D reactivity of Del-red blood cells. Transfusion Med., 26: 123-129.

Chen, Q., M. Li, X.S. Lu, R. Lu and J. Sun et al., 2012. Molecular basis of weak D and DEL in Han population in Anhui Province, China. Chin. Med. J., 125: 3251-3255.

Cruz, B.R., A.K. Chiba, E. Moritz and J.O. Bordin, 2012. RHD alleles in Brazilian blood donors with weak D or D-negative phenotypes. Transfusion Med., 22: 84-89.

Flegel, W.A., 2011. Molecular genetics and clinical applications for RH. Transfus. Apher. Sci., 44: 81-91.

Gupta, A., S. Mirza, S. Khurana, R. Singh and S. Chaturvedi et al., 2016. Enigmatic weak D antigen: An experience in a tertiary care hospital of East Delhi. J. Clin. Diagn. Res., 10: EC12-EC15.

He, J., Y. Ying, X. Hong, X. Xu, F. Zhu and H. Lv, 2015. Molecular basis and zygosity determination of $D$ variants including identification of four novel alleles in Chinese individuals. Transfusion, 55: 138143.

ISBT., 2016. Red cell Immunogenetics and blood group terminology. Maharana Pratap Inter State Bus Terminus, Delhi, India. http://www.isbtweb.org/working-parties/red-cellimmunogenetics-and-blood-group-terminology/

Kyaw, T.Z., J.A. Maniam, V.S. Nadarajan and H. Shanmugam, 2012. Molecular analysis of Malaysian Chinese D-donors: A single centre experience. Asian Biomed., 6: 27-33.

Lin, I.L., M.C. Shih, M.H. Hsieh, T.C. Liu and S.E. Chang et al., 2003. Molecular basis of weak D in Taiwanese. Annl. Hematol., 82: 617-620.

Luettringhaus, T.A., D. Cho, D.W. Ryang and W.A. Flegel, 2006. An easy RHD genotyping strategy for D-East Asian persons applied to Korean blood donors. Transfusion, 46: 2128-2137.

Sandler, S.G., W.A. Flegel, C.M. Westhoff, G.A. Denomme and M. Delaney et al., 2015. It's time to phase in RHD genotyping for patients with a serologic weak D phenotype. Transfusion, 55: 680689. 
Shao, C.P., J.H. Maas, Y.Q. Su, M. Kohler and T.J. Legler, 2002. Molecular background of Rh D-positive, Dnegative, Del and weak D phenotypes in Chinese. Vox Sanguinis, 83: 156-161.

Virk, M. and S. G. Sandler, 2015. Rh Immunoprophylaxis for women with a serologic weak D phenotype. Lab. Med., 46: 190-194.

Wafi, M.E., H.E. Housse, N. Nourichafi, K. Bouisk and M. Benajiba et al., 2016. Prevalence of weak D phenotype among $\mathrm{D}$ negative $\mathrm{C} / \mathrm{E}+$ blood donors in Morocco. Int. J. Blood Transfusion Immunohematology, 6: 3-7.
Wagner, F.F., C. Gassner, T.H. Muller, D. Schonitzer and F. Schunter et al., 1999. Molecular basis of weak D phenotypes. Blood, 93: 385-393.

Yan, L., J. Wu, F. Zhu, X. Hong and X. Xu, 2007. Molecular basis of $\mathrm{D}$ variants in Chinese persons. Transfusion, 47: 471-477.

Zacarias, J.M.V., D.F.E.M. Pereira, J.E.L. Visentainer, G.A.S. Guelsin and D.F.C. Melo et al., 2016. Frequency of RHD variants in Brazilian blood donors from Parana State, Southern Brazil. Transfusion Apheresis Sci., 55: 120-124. 